\title{
Assessment of lactoferrin-conjugated solid lipid nanoparticles for efficient targeting to the lung
}

\author{
Satish Shilpi • Vishnoo Dayal Vimal • \\ Vandana Soni
}

Received: 7 August 2014/ Accepted: 16 February 2015/Published online: 27 March 2015

(C) The Author(s) 2015. This article is published with open access at Springerlink.com

\begin{abstract}
The aim of the present study was to develop a target oriented drug delivery system for the lungs. Lactoferrin (Lf)-coupled solid lipid nanoparticles (SLNs) bearing rifampicin was prepared by a solvent injection method. The prepared nanoparticles were characterized for shape, particle size, polydispersity and percentage drug entrapment. An optimized formulation was then studied for its in vivo performance in animals and to determine its targeting efficiency. It was observed that, upon coupling with Lf, the size of SLNs increased while the percent entrapment efficiency decreases. In in vitro release, determined by a dialysis technique, analysis showed that uncoupled SLNs exhibited higher drug release as compared to coupled SLNs. An in vivo biodistribution study shows $47.7 \pm 0.4$ drug uptakes by the lungs, which was 3.05 times higher in comparison to uncoupled SLNs. These biodistribution studies are further supported by the fluorescence study that revealed enhanced uptake of Lf-coupled SLNs in the lung. From the presented results, it can be concluded that Lfcoupled SLNs enhanced drug uptake in the lung. Moreover, lactoferrin is an efficient molecule that can be used for targeting active agents directly to the lung.
\end{abstract}

\footnotetext{
S. Shilpi $(\bowtie) \cdot$ V. D. Vimal · V. Soni

Department of Pharmaceutical Sciences, Dr. Hari Singh Gour

University, Sagar 470 003, Madhya Pradesh, India

e-mail: shilpisatish@gmail.com

V. D. Vimal

e-mail: satish_shilpi@yahoo.co.in

V. Soni

e-mail: drvandanasoni@gmail.com

S. Shilpi

Ravishankar College of Pharmacy,

Bhopal 462 010, Madhya Pradesh, India
}

Keywords Biodistribution - Lung targeting - SLNs · Drug delivery $\cdot$ Lactoferrin

\section{Introduction}

The success of novel drug delivery systems depends on the development of formulations that are capable of improving the therapeutic index of biologically active molecules by increasing their concentration specifically at desired target sites or organs. It is well known that various novel carriers have been used for drug delivery to the lungs for the treatment of tuberculosis, for example, microparticle (Zhou et al. 2005; Zhuang et al. 2012) poly(lactic-co-glycolic acid) (PLGA) nanospheres (Tomoda et al. 2005; Tomoda and Makino 2007), stealth liposomes (Deol and Khuller 1997), functionalized nanoparticles (Sharma et al. 2004), and SLNs (Anisimova et al. 2000; Pandey et al. 2005; Pandey and Khuller 2005). Microparticles offer excellent aerodynamic properties and their large geometric size reduces their uptake by alveolar macrophages, making them a suitable carrier for sustained drug release in the lungs. Similarly, nanocarriers offers significant potential for prolonged drug release in the lungs because they largely escape uptake by lung-surface macrophages and can remain in the pulmonary tissue for a long time. Conjugation of drugs to polymers as polyethylene glycol or stealth liposomes can be particularly beneficial for sustaining the release of drugs. Liposomes can be prepared with lipids endogenous to the lungs and are particularly safe. Their composition can be adjusted to modulate drug release and they can encapsulate both hydrophilic and lipophilic compounds with high drug loading (Loira-Pastoriza et al. 2014).

Among the above, SLNs may be an alternative drug carrier system that offers several advantages conferred by 
their colloidal dimensions, including: easy incorporation of both lipophilic and hydrophilic drugs; improved biocompatibility; flexible surface functionality; nanoscopic structure; monodispersity; and high encapsulation efficiency (Muller and Keck 2010; Alpiaz et al. 2008; Carneiro et al. 2012; Jaspart et al. 2005; Shuhendler et al. 2012; Wang et al. 2012; Yang et al. 2012).

It is well known that the targeting of the lung offers a challenge due to the mucocilliary clearance. In this context, the ligand-anchored drug delivery system proves its potential in achieving enhanced site-specific drug delivery as well as reduced reticular endothelial system (RES) uptake (Sahu et al. 2014; Baek and Cho 2013; Makino et al. 2004). However, the clinical success of such an approach depends on the selection of appropriate ligands lacking immunogenic potential with the ability to mediate cargo internalization by the target cell (Allen 2002). With this concern in mind, lactoferrin (Lf) may function as a ligand suitable for coupling with SLNs, generating a promising drug delivery system to the lungs.

Lactoferrin is an $80-\mathrm{kDa}$ iron-binding glycoprotein of the transferrin family. Lf is thought to play a role in innate defense and exhibits a diverse range of biological activities, including antimicrobial, antiviral, antioxidant, immunomodulation, modulation of cell growth, and binding and inhibition of several bioactive compounds such as lipopolysaccharide and glycosaminoglycan. Bovine Lf has been found to significantly inhibit colon, esophagus, lung and bladder carcinogenesis in rats when administered orally in the post-initiation stages (Tsuda et al. 2002). Lf receptors are expressed on the apical surface of bronchial epithelial cells and this conception is utilized to achieve targeted drug delivery to the lungs (Elfinger et al. 2007).

To achieve site specificity, Lf was used as a ligand in present project. Chemical cross linking strategies were utilized for the conjugation of Lf with SLNs and the $-\mathrm{NH}_{2}$ group present at the surface of stearylamine containing SLNs conjugated with the $-\mathrm{COOH}$ groups of Lf. Lf-coupled SLN enhances drug delivery to the lungs because the receptors of this protein are over expressed in the lungs (Schubert and Goymann 2003). Hence, this system may become a promising tool for enhancing drug delivery to treat lung-associated diseases.

Recently, multiple drug chemotherapy has formed the backbone of antituberculotic therapy. Particularly, rifampicin is the first choice drug in the treatment of tuberculosis. But the current treatment of tuberculosis involves prolonged oral administration of large systemic doses of combined antibiotics that are associated with unwanted side effects and poor patient compliance (Ito and Makino 2004; O'Hara and Hickey 2000).

Therefore, the current work was aimed to design and evaluate the efficacy of Lf-anchored rifampicin loaded
SLNs for effective management of tuberculosis. In this project Lf was conjugated on the surface of SLN, in which the $-\mathrm{NH}_{2}$ group of stearylamine containing $\mathrm{SLN}$ were conjugated with the $-\mathrm{COOH}$ groups of Lf. Lf-coupled SLNs enhance lung cancer cell specific (lactoferrin receptor overexpressing cells) targeting and drug delivery. The present study reveals the targeting potential of Lf-coupled SLNs for site-specific delivery of rifampicin to the lungs.

\section{Materials}

The drug (rifampicin) was obtained as a gift sample from Park Pharmaceuticals, Panchkula. Tristearin, soya lecithin, stearylamine, Triton X-100, Tween 80, Sephadex G-50 and 1-ethyl-3-(3-dimethylaminopropyl) carbodiimide hydrochloride (EDC) were purchased from Sigma Chemicals (St Louis, MO, USA). Dialysis membranes (molecular weight cut off 3500) were purchased from Himedia, Mumbai, India. All other reagents and solvents were of analytical grade.

Preparation of solid lipid nanoparticles (SLNs)

\section{Preparation of the uncoupled SLNs}

SLNs were prepared according to the solvent injection method previously developed by Schubert and Goymann (2003). In this method, tristearin (100 mg), soya lecithin $(100 \mathrm{mg})$ and stearylamine $(10 \mathrm{mg})$ were dissolved in a minimum quantity of absolute alcohol in different ratios and heated to about $50{ }^{\circ} \mathrm{C}$. Tween $80(0.5 \% \mathrm{v} / \mathrm{v})$ containing phosphate buffer $(\mathrm{pH} 7.4)$ solution was heated separately at the same temperature and was used as the aqueous phase. Then the organic phase containing a lipid mixture was added using a preheated syringe to an aqueous solution at the same temperature with continuous stirring for a definite time period. The lipid suspension was then sonicated by probe sonicator of an $10 \phi$-amplitude lever at a 20 -s pulse rate (power output $2 \mathrm{KW}$ ) and a $20-\mathrm{kHz}$ frequency for $120 \mathrm{~s}$, which gives uniformity in the size of the SLNs. Drug-loaded SLNs were prepared by the same procedure in which the drug $(10 \mathrm{mg})$ was dissolved in phosphate-buffered saline (PBS; $\mathrm{pH} 7.4$ ) to obtain a 10:100 drug:lipid ratio. The complete methodology for preparation of SLNs was shown in Fig. 1.

\section{Conjugation of SLNs with lactoferrin (Lf)}

Coupling of SLNs with Lf was performed according to the method reported by Gupta et al. (2007) via carbodiimide chemistry (Wissing and Muller 2002), i.e., coupling of the 
Fig. 1 Schematic diagram for SLN preparation

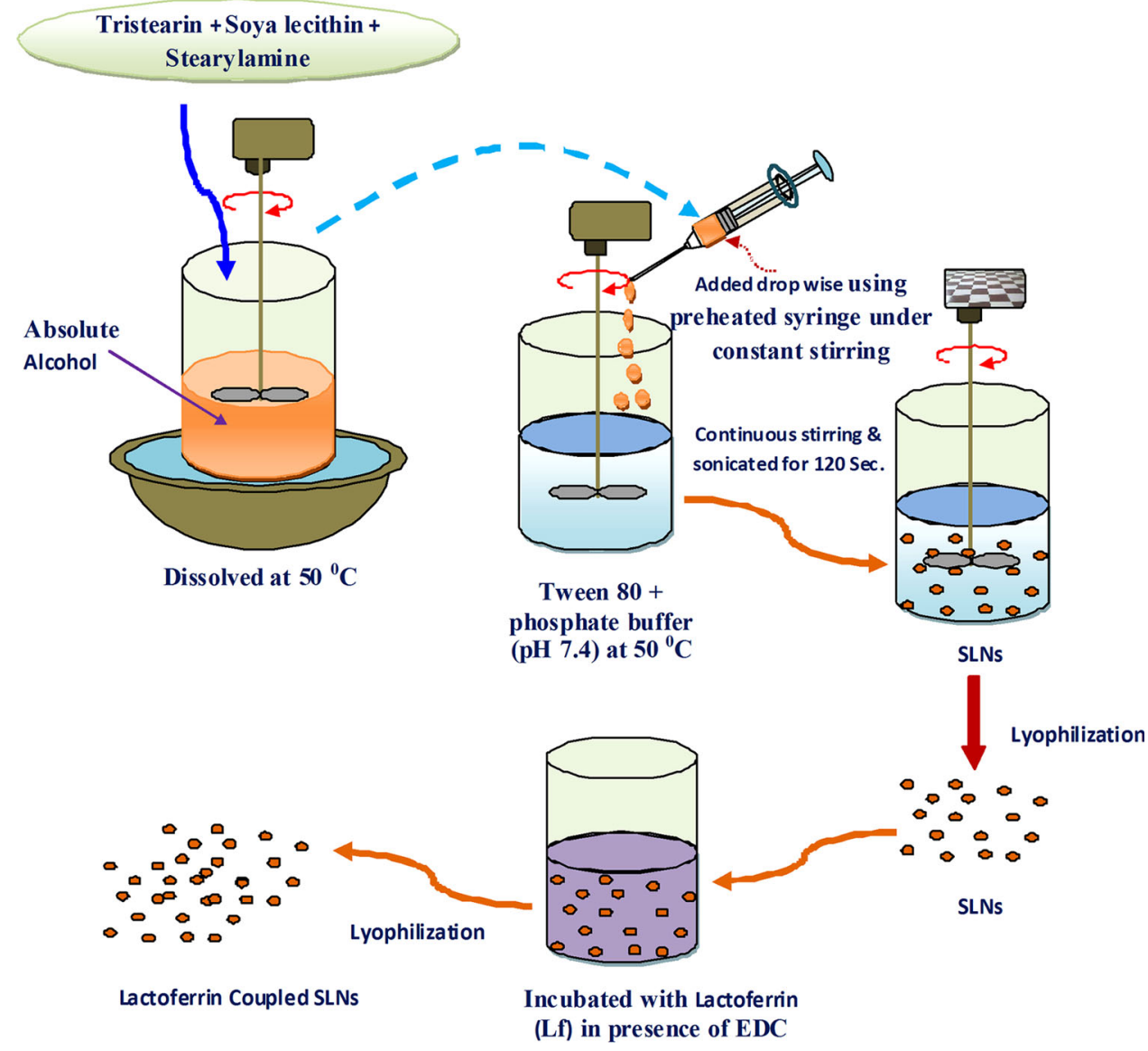

Table 1 Various parameters of uncoupled and coupled SLNs bearing rifampicin

\begin{tabular}{|c|c|c|c|c|c|c|}
\hline S. No. & Formulation & Particle size $(\mathrm{nm})$ & PDI & Zeta potential $(\mathrm{mV})$ & Entrapment efficiency (\%) & Coupling efficiency (\%) \\
\hline 1 & Uncoupled SLNs & $235 \pm 2$ & 0.076 & $22 \pm 1$ & $73.4 \pm 3$ & Nil \\
\hline 2 & Lactoferrin-coupled SLNs & $271 \pm 2$ & 0.124 & $23 \pm 2$ & $68.4 \pm 2$ & 22.7 \\
\hline
\end{tabular}

Mean $(n=3) \pm \mathrm{SD}$

Lf carboxylic group with the stearylamine amine group present on the surface of the previously formed drugloaded SLNs in the presence of $N$-ethyl- $N$-(dimethylaminopropyl)-carbodiimide (EDC). In preparation of coupled SLNs, the drug-loaded formulations (100 mg) were suspended in a PBS $(\mathrm{pH} 7.4 ; 10 \mathrm{ml})$ containing Lf $(10 \mathrm{mg})$ and EDC (10 mg) and then incubated for $2 \mathrm{~h}$ at room temperature. Plain SLNs were removed by passing the formulation through a Sephadex G-50 mini column.

Characterization of uncoupled and coupled SLNs

\section{Particle size, polydispersity index and zeta potential}

The average particle size and polydispersity index (PDI) of the SLNs were determined by photon correlation spectroscopy using a Zetasizer DTS, version 4.10 (Malvern Instrument, UK). The formulations were diluted to $1: 9 \mathrm{v} / \mathrm{v}$ with deionized water. The particles size and PDI were represented by the average diameter of the Gaussian distribution function in the logarithmic axis mode. Particle size, size distribution and zeta potential of the SLN formulation was performed at the National Institute of Pharmaceutical Education and Research (NIPER), Mohali, India.

Surface charge measurement of the SLNs was based on the zeta potential $(\varepsilon)$ that was calculated according to Helmholtz-Smoluchowsky from their electrophoretic mobility. For measurement of zeta potential, a Zetasizer was used with a field strength of $20 \mathrm{~V} / \mathrm{cm}$ on a large bore measures cell. Samples were diluted with $0.9 \% \mathrm{NaCl}$ adjusted to a conductivity of $50 \mu \mathrm{S} / \mathrm{cm}$ (Nassimi et al. 2010). The results are given in Table 1. 
Shape and surface morphology

In order to examine the SLN surface morphology, the formulations were viewed via scanning electron microscopy (SEM). SEM samples were prepared by lightly sprinkling the lyophilized nanoparticle powder on a double adhesive tape stuck on an aluminum stub. The stubs were then coated with gold to a thickness of about $300 \AA$ using a

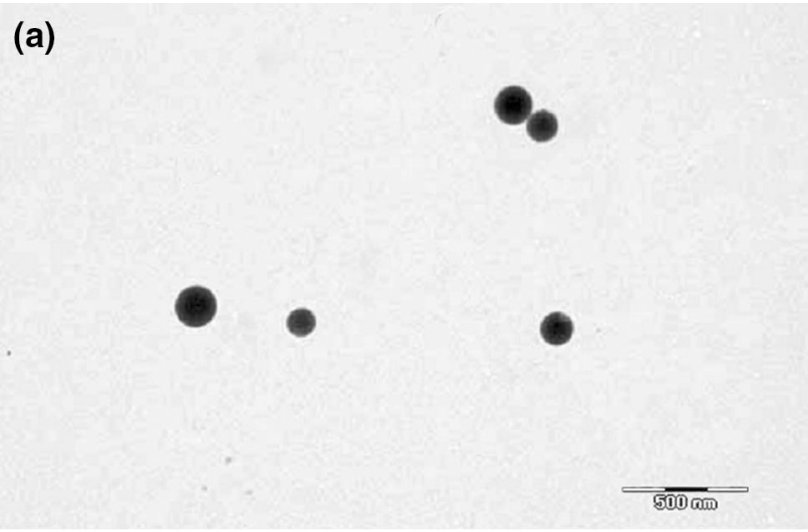

(b)

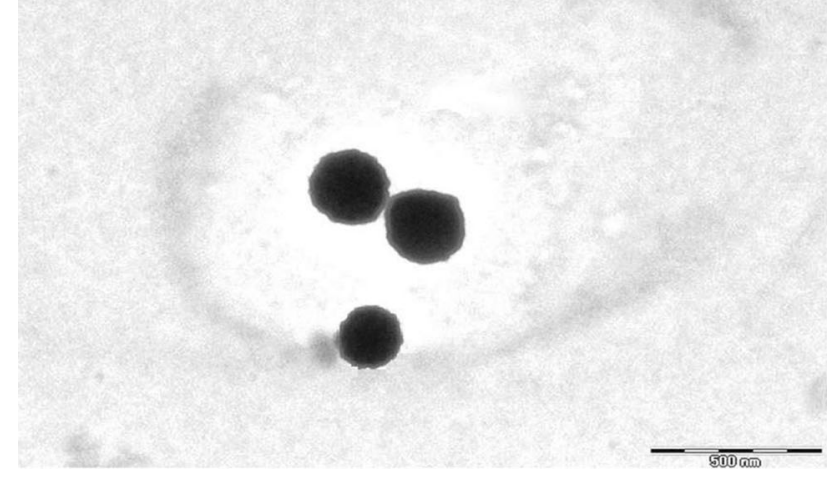

Fig. 2 a TEM microphotograph of uncoupled SLNs. b TEM microphotograph of Lf-coupled SLNs

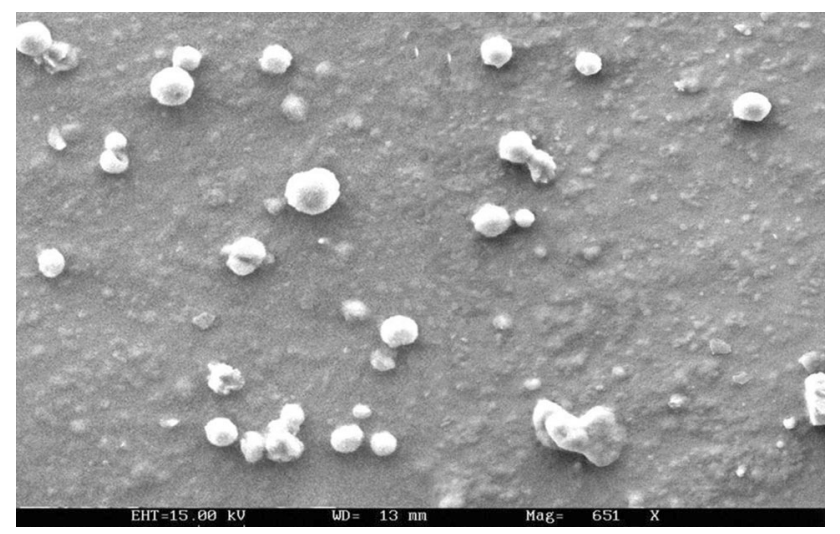

Fig. 3 SEM microphotograph of coupled SLNs sputter coater. The photomicrographs were taken with a scanning electron microscope (JEOL JSM-6100). Transmission electron microscopy (TEM; Philips CM12 Electron Microscope, Eindhoven, Netherlands) at an acceleration voltage of $20 \mathrm{kV}$ was used to visualize nanoparticles. Samples were negatively stained with $2 \%$ aqueous solution of phosphotungstic acid. TEM images of uncoupled and coupled Lf are presnted in Fig. 2a, b, respectively. SEM images (Fig. 3) show Lf-coupled SLNs. SEM and TEM were performed at the All India Institute of Medical Sciences (AIIMS), New Delhi.

\section{Entrapment efficiency}

Entrapment efficiency of uncoupled and coupled SLNs was determined using the method described by Gupta et al. (2007) and Fry (1978). The drug not entrapped was removed from the SLNs by passing the formulation through a Sephadex G-50 minicolumn. The weighed amount of Sephadex G-50 was properly mixed with sufficient amount of distilled water in a beaker and kept for $24 \mathrm{~h}$ for complete swelling. After complete swelling, Sephadex dispersion was placed in a 1-ml PVC syringe (Dispovan) packed with glass wool and a small piece of Whatman filter paper at the bottom end to provide stability for the Sephadex column at $3,000 \mathrm{rpm}$. The amount of drug not entrapped in the SLNs was determined by passing the formulation from the Sephadex column, centrifuging at 3,000 rpm, and collecting the elution using the equation from Gupta et al. (2007). After removing the un-entrapped drugs, the SLNs were collected and lysed using $1 \%$ Triton X100; drug entrapment was then analysed spectroscopically.

\section{$\%$ Drug entrapment \\ $=\frac{\text { Theoretical drug content }- \text { Practical drug content }}{\text { Theoretical drug content }} \times 100$}

This solution was then diluted ten times with PBS ( $\mathrm{pH}$ 7.4) and analyzed with a spectrophotometer at $\lambda_{\max }$ of

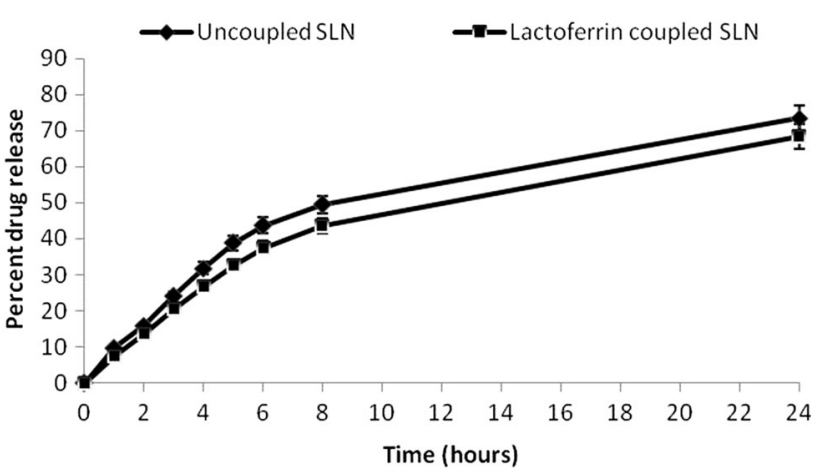

Fig. 4 In vitro drug release of uncoupled and coupled SLNs 
$476 \mathrm{~nm}$. The same procedure was applied for determining drug entrapment of the Lf-coupled SLNs. The percentages of drug entrapment in uncoupled and coupled SLNs are recorded in Table 1.

\section{In vitro drug release}

The drug release of SLNs and Lf-coupled SLNs was performed in PBS ( $\mathrm{pH}$ 7.4) using the dialysis bag technique. The dialysis bag retains nanoparticles and allows the free drug into the dissolution media with a molecular weight cut off point 3.5 KD. The bags were soaked in double-distilled water for $12 \mathrm{~h}$ before use. One $\mathrm{ml}$ of pure SLN formulation containing about $100 \mathrm{mg}$ of the drug in about $150 \mathrm{mg}$ of SLN free of any unentrapped drug was taken in a dialysis bag and placed in a beaker containing $50 \mathrm{ml}$ of $\mathrm{PBS}(\mathrm{pH} 7.4)$ at $37 \pm 1{ }^{\circ} \mathrm{C}$ throughout the study. The samples were withdrawn after specified time intervals and replaced with the same volume of PBS (pH 7.4). The withdrawn samples were analyzed for drug content by spectrophotometer at $476 \mathrm{~nm}$ (Fig. 4).

\section{Coupling efficiency}

The Lf concentration in the coupled SLNs was determined by the Bradford method of protein estimation with minor modifications (Calleja et al. 2004). Briefly, $1 \mathrm{ml}$ of Lfcoupled SLNs containing about $100 \mathrm{mg}$ of SLN formulation was placed in a volumetric flask with $1 \mathrm{ml}(10 \%)$ Coomassie blue $\mathrm{G}$ dye solution, and the volume was adjusted to $10 \mathrm{ml}$ with distilled water. To determine the $\mathrm{Lf}$ concentration, the absorbance at $595 \mathrm{~nm}$ was measured and compared with a blank containing the same amount of dye.

In vivo organ distribution study

Fasted albino rats (average weight 150-200 gm) were divided into three groups each containing 12 animals. Animals of the first group were kept as a control that received an aqueous solution of free drug, while the second and third groups received uncoupled and coupled formulations, respectively. A dose of $1.5 \mathrm{mg} / \mathrm{kg}$ body weight was given to the rats intravenously. All studies were carried out according to the guidelines of the Council for the Purpose of Control and Supervision of Experiments on Animals (CPCSEA), Ministry of Social Justice and Empowerment, Government of India and approved by the University Animal Ethical Committee, Sagar (MP), India and performed at the Department of Pharmaceutical Sciences, Dr. H. S. Gour University, Sagar, India.
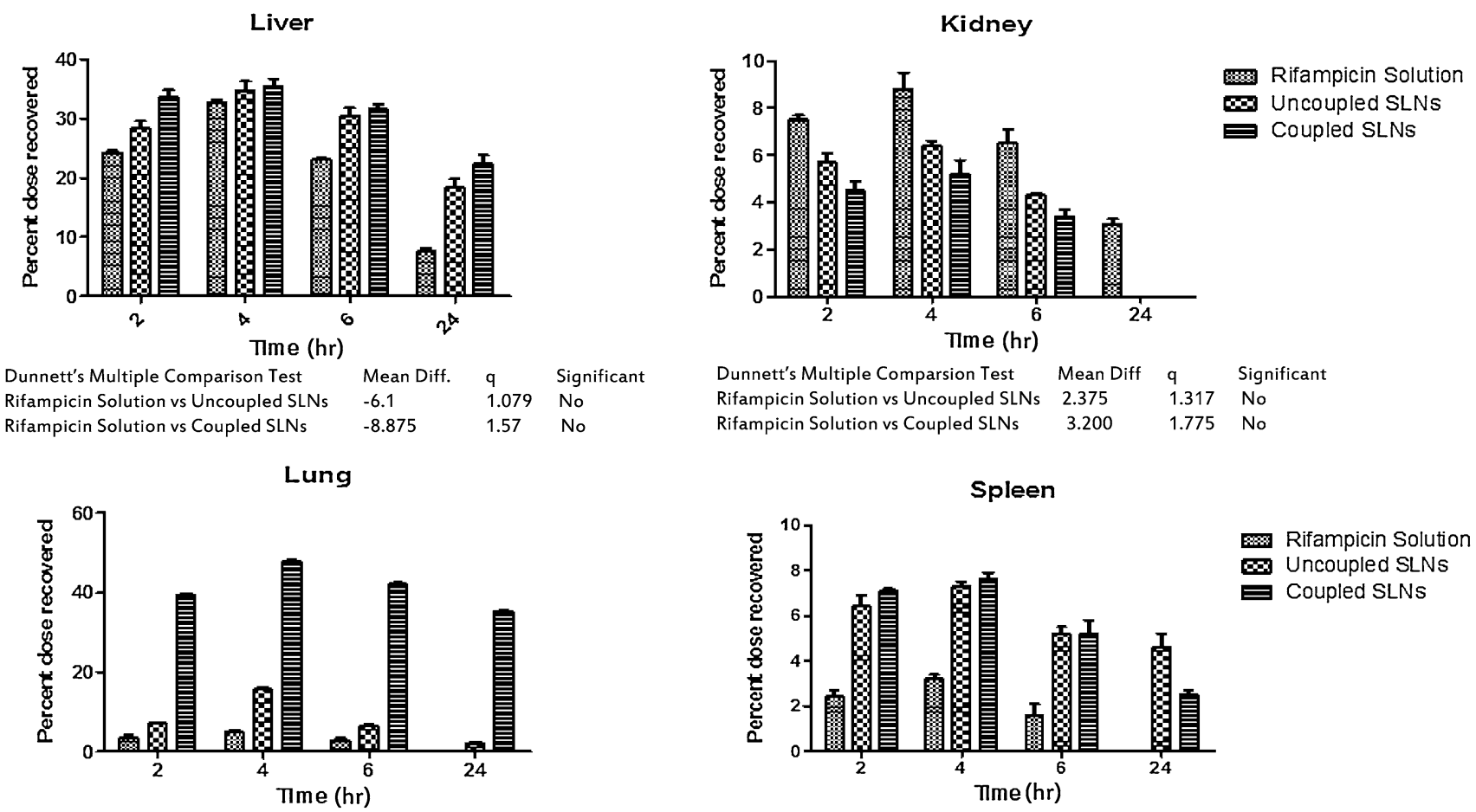

$\begin{array}{llll}\text { Dunnett's Multiple Comparsion Test } & \text { Mean Diff } & \text { q } & \text { Significant } \\ \text { Rifampicin Solution vs Uncoupled SLNs } & -4.975 & 1.532 & \text { No } \\ \text { Rifampicin Solution vs Coupled SLNs } & -38.25 & 11.78 & \text { Yes }(p-<0.001)\end{array}$

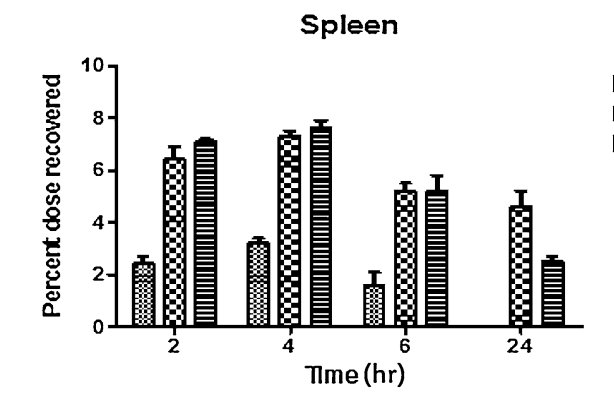

Dunnett's Multiple Comparsion Test Mean Diff q Significant

Rifampicin Solution vs Uncoupled SLNs $\quad-4.075 \quad 3.39 \quad$ Yes $(p-<0.05)$

Rifampicin Solution vs Coupled SLNs $\quad-3.8 \quad 3.161$ Yes $(\mathrm{p}-<0.05)$

요 Rifampicin Solution

$\infty$ Uncoupled SLNs

— Coupled SLNs

Fig. 5 Percent dose recovered in different organs from various formulations, consists of statistically significant values 
After administration of the formulations, rats from each group were sacrificed after 2, 4, 6 and $24 \mathrm{~h}$. One gram of each organ was homogenized with $2 \mathrm{ml}$ of PBS ( $\mathrm{pH} 7.4$ ) using a homogenizer. In the case of organs weighing $<1 \mathrm{~g}$, the whole organ was used and the amount of drug present in each organ and blood sample was determined by using the high performance liquid chromatography (HPLC) method reported by Calleja et al. (2004) (Fig. 5) (Calleja et al. 2004; Kar 2005). The mobile phase consisted of water ( $\mathrm{pH} 2.27$ adjusted with orthophosphoric acid)-acetonitrile $(40: 60, \mathrm{v} / \mathrm{v})$ at a flow-rate of $1 \mathrm{ml} / \mathrm{min}$. Chromatography was carried out at $25{ }^{\circ} \mathrm{C}$ and the elute was monitored at $333 \mathrm{~nm}$ on a $\mathrm{C}_{18}(4.6 \times 250 \mathrm{~mm}, 5 \mu \mathrm{m})$ column with a UV detector.

\section{Estimation of rifampicin in blood plasma}

Blood was collected through a cardiac puncture in a centrifuge tube containing heparin (anticoagulant) and centrifuged at 2,000 rpm for $15 \mathrm{~min}$. Acetonitrile $(1 \mathrm{ml} / \mathrm{ml})$ was added to the in supernatant to precipitate the proteins; the solution was then centrifuged at 2,000 rpm for $15 \mathrm{~min}$ and supernatant was collected a second time. The supernatant was filtered through a $0.45-\mu \mathrm{m}$ membrane filter and analysed as per the procedure used for the organ distribution study. Estimation of rifampicin was done by HPLC as described above and is shown graphically in Fig. 6.

\section{Fluorescence microscopy}

Fluorescence microscopy was performed in order to confirm the lung uptake of the Lf-coupled SLNs. Rhodamine 6G was used as the fluorescence marker, which was encapsulated into uncoupled and coupled SLNs. The formulations were administered intravenously and sacrificed after $60 \mathrm{~min}$; lungs were then excised and isolated. They were cut into small pieces and washed in Ringer's solution with subsequent drying using tissue paper. Dried pieces of

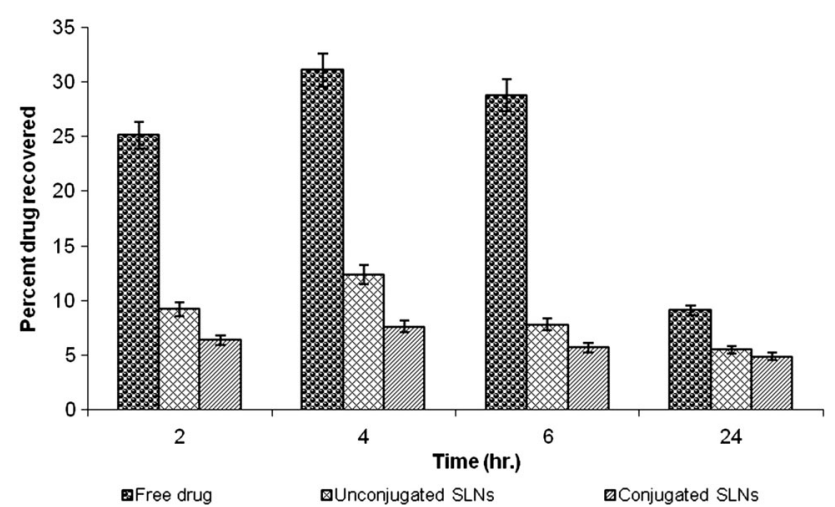

Fig. 6 Percent drug recovered in blood plasma from various formulations various organs were fixed in Carnay's fluid (absolute alcohol:chloroform:glacial acetic acid, 3.5:1:0.5). Then, microtomy was done and ribbons of the sections obtained were fixed on the slides using egg albumin solutions as fixative. The sections were viewed under fluorescence microscope and their photomicrographs are shown in Fig. 7.

\section{Statistical analysis}

Data are expressed as the mean \pm standard deviation (SD) and statistical analysis was carried out employing the oneway analysis of variance (ANOVA) test using the PRISM software (Graph Pad). A value of $P<0.005$ was considered statistically significant (Fig. 5).

Results and discussion

Lf-coupled and uncoupled SLNs were prepared using tristearin, soya lecithin and stearylamine to improve Rifampicin transport to the lungs, and optimized for size, shape and percent entrapment efficiency. Coupling of optimized SLN formulations with Lf was performed using carbodiimide chemistry in which the carboxylate group of Lf conjugated with the amine group of stearylamine present on the surface of previously formed drug-loaded SLNs, with help of EDC as a coupling agent. The coupling of Lf with the SLNs was performed via carbodiimide chemistry; the -CONH-(amide linkage) bond is formed between the $-\mathrm{NH}_{2}$ groups present on the surface of the SLNs (due to the presence of stearylamine) and the -COOH group of the Lf. In FTIR spectra, the characteristic peak at $3,288 \mathrm{~cm}^{-1}$ represents $-\mathrm{N}-\mathrm{H}$ stretching of the primary amine, and the peak at $1,625 \mathrm{~cm}^{-1}$ represents $-\mathrm{C}-\mathrm{O}$ stretching. The disappearance of the secondary amine peak in the spectra was additional confirmation of lactoferrin conjugation (Fig. 8).

TEM and SEM studies revealed the spherical shape of coupled and uncoupled SLNs (Figs. 2a, b, 3), whereas the mean diameter of Lf-coupled and uncoupled SLNs was found to be $235 \pm 2$ and $271 \pm 2 \mathrm{~nm}$, respectively (Table 1). The size of the coupled formulation was found to be higher when compared to the uncoupled formulation, which could be due to the coupling of Lf on the surface of the SLN. The PDI was found to be $<0.2$ for both formulations, indicating a narrow size distribution of particles and, consequently, a uniform distribution. The zeta potential of uncoupled and coupled SLNs was found to be $22 \pm 1$ and $23 \pm 1 \mathrm{mV}$, respectively (Table 1 ). The zeta potential of both formulations was found to be similar, which indicates that Lf does not markedly affect the cationic surface charges of SLNs. The drug entrapment efficiency of Lf-SLNs was found to be less as compared to 
Fig. 7 Fluorescence photomicrographs after administration of uncoupled (a) and coupled SLNs (b)
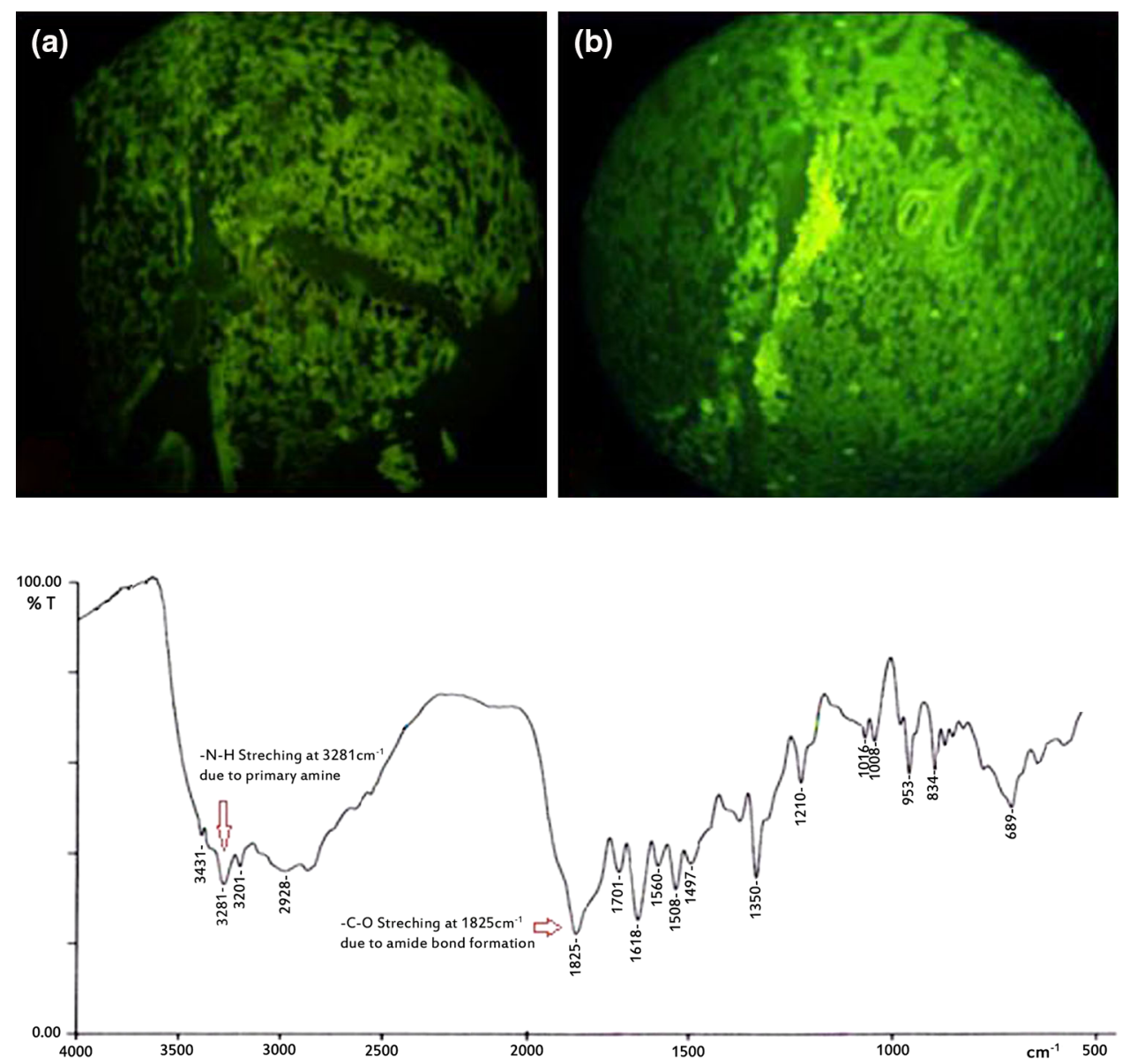

Fig. 8 FTIR spectra of lactoferrin-conjugated SLNs drug in various organs. However, the concentration of the drug in plasma after administration of different types of SLNs (uncoupled and coupled) was considerably low. For the uncoupled formulation, the maximum percent dose recovered in plasma was found to be $12.4 \pm 0.2 \%$ after $4 \mathrm{~h}$, which decreased to $5.5 \pm 0.4 \%$ after $24 \mathrm{~h}$. For the coupled formulation, the maximum percent dose recovered in plasma was found to be $7.6 \pm 0.7 \%$ after $4 \mathrm{~h}$, which decreased to $4.9 \pm 0.2 \%$ after $24 \mathrm{~h}$ (Fig. 5). The significant reduction in drug concentration in blood plasma may be due to the fact that most of the drug present in blood was entrapped in SLNs, leading to a reduced rifampicin toxicity; also, the method by which the drug was extracted from the plasma did not extract the drug from the SLNs.

The lung uptake of both coupled and uncoupled SLNs was assessed via a biodistribution study after intravenous administration of a solution of free drug in coupled and uncoupled formulations. The results of the biodistribution studies exhibit a maximum accumulation of the drug in the liver, kidney and spleen after intravenous administration of a plain drug. The concentrations of drug accumulated in different organs following intravenous administration of a plain drug after $4 \mathrm{~h}$ were found to be $32.7 \pm 0.5 \%$ in the drug from the kidney and simultaneous distribution of the 
Table 2 Organ drug distribution from various formulations

\begin{tabular}{|c|c|c|c|c|c|}
\hline & \multirow[t]{2}{*}{ Time (h) } & \multicolumn{4}{|c|}{ Percent dose recovered } \\
\hline & & Liver & Kidney & Lung & Spleen \\
\hline \multirow[t]{4}{*}{ Rifampicin drug solution } & 2 & $24.3 \pm 0.3$ & $7.5 \pm 0.2$ & $3.6 \pm 0.7$ & $2.4 \pm 0.3$ \\
\hline & 4 & $32.7 \pm 0.5$ & $8.8 \pm 0.7$ & $4.9 \pm 0.4$ & $3.2 \pm 0.2$ \\
\hline & 6 & $23.1 \pm 0.2$ & $6.5 \pm 0.6$ & $2.9 \pm 0.4$ & $1.6 \pm 0.5$ \\
\hline & 24 & $7.6 \pm 0.5$ & $3.1 \pm 0.2$ & Nil & Nil \\
\hline \multirow[t]{4}{*}{ Uncoupled SLNs } & 2 & $28.4 \pm 1.2$ & $5.7 \pm 0.4$ & $7.1 \pm 0.3$ & $6.4 \pm 0.5$ \\
\hline & 4 & $34.8 \pm 1.6$ & $6.4 \pm 0.2$ & $15.6 \pm 0.6$ & $7.3 \pm 0.2$ \\
\hline & 6 & $30.5 \pm 1.4$ & $4.3 \pm 0.1$ & $6.5 \pm 0.2$ & $5.2 \pm 0.3$ \\
\hline & 24 & $18.4 \pm 1.5$ & Nil & $2.1 \pm 0.5$ & $4.6 \pm 0.6$ \\
\hline \multirow[t]{4}{*}{ Coupled SLNs } & 2 & $33.7 \pm 1.2$ & $4.5 \pm 0.4$ & $39.3 \pm 0.5$ & $7.1 \pm 0.1$ \\
\hline & 4 & $35.5 \pm 1.3$ & $5.2 \pm 0.6$ & $47.7 \pm 0.4$ & $7.6 \pm 0.3$ \\
\hline & 6 & $31.6 \pm 0.8$ & $3.4 \pm 0.3$ & $42.1 \pm 0.4$ & $5.2 \pm 0.6$ \\
\hline & 24 & $22.4 \pm 1.5$ & Nil & $35.3 \pm 0.2$ & $2.5 \pm 0.2$ \\
\hline
\end{tabular}

$\mathrm{SD} \pm$ mean $(n=3)$

coupled SLNs were compared with uncoupled SLNs (Fig. 5).

Fluorescence photomicrographs (Fig. 7) showed the qualitative uptake and the localization pattern of SLNs in the lung. The Lf-coupled SLNs were loaded with rhodamine $6 \mathrm{G}$ and were administered intravenously to albino rats; the lung was then isolated after $60 \mathrm{~min}$ from a sacrificed animal and photomicrographs were taken. The alveolar macrophages were distinctly filled with the formulation, while other cells, blood capillaries and thick junction places depict diffuse fluorescence as shown in the photomicrograph. The photomicrographs (Fig. 7b) clearly show access of the Lf-coupled SLNs into the lung.

\section{Conclusion}

The aim of the study was to design SLNs loaded with the anti-tuberculosis drug rifampicin. One factor determing the success of a drug delivery system is a higher concentration of the drug at the site of action with decreased side effects to the non-target tissues. Prepared SLNs were coupled with Lf to achieve target-specific delivery of rifampicin to the lungs. In vitro drug release and biodistribution studies of rifampicin-loaded coupled and uncoupled Lf showed that the Lf-coupled formulation sustains the drug release as well as delivers the drug at a higher concentration to the target. Based on these results, it can be concluded that the formulations developed in this work may be considered as effective drug delivery systems for the treatment of tuberculosis and other lung-associated diseases.This work, however, requires further experimental study.

Acknowledgments The authors are grateful to Park Pharmaceuticals, Panchkula for providing a gift sample of the drug, the Anatomy Division at the AIIMS, New Delhi for providing the necessary facility 
for conducting TEM and SEM, and the NIPER, Chandigarh for zeta potential data. This work was financially supported by the All India Council for Technical Education (AICTE), India.

Conflict of interest The authors report no conflict of interest. The authors are soley responsible for the content and writing of this paper.

Open Access This article is distributed under the terms of the Creative Commons Attribution License which permits any use, distribution, and reproduction in any medium, provided the original author(s) and the source are credited.

\section{References}

Allen TM (2002) Ligand targeted therapeutic in anticancer therapy. Nat Rev 2:263

Alpiaz A, Mezzena M, Scatturin A, Scalia S (2008) Solid lipid microparticles for the stability enhancement of the polar drug $N$ 6-cyclopentyladenosine. Int J Pharm 355(1-2):81-86

Anisimova YV, Gelperina SI, Peloquin CA, Heifets LB (2000) Nanoparticles as antituberculosis drugs carriers: effect on activity against Mycobacterium tuberculosis in human monocyte-derived macrophages. J Nanopart Res 2:165-171

Baek JS, Cho CW (2013) 2-Hydroxypropyl- $\beta$-cyclodextrin-modified SLN of paclitaxel for overcoming $p$-glycoprotein function in multidrug-resistant breast cancer cells. J Pharm Pharmacol 65(1):72-78

Calleja I, Blanco-Prieto MJ, Ruz N, Renedo MJ, Dios-Vieitez MC (2004) High-performance liquid-chromatographic determination of Rifampicin in plasma and tissues. J Chromatogr A 1031:289-294

Carneiro G, Silva EL, Pacheco LA, de Souza-Fagundes EM, Corrêa NC, de Goes AM et al (2012) Formation of ion pairing as an alternative to improve encapsulation and anticancer activity of all-trans retinoic acid loaded in solid lipid nanoparticles. Int $\mathbf{J}$ Nanomed 7:6011-6020

Deol P, Khuller GK (1997) Lung specific stealth liposomes: stability, biodistribution and toxicity of liposomal antitubercular drugs in mice. Biochim Biophys Acta 1334:161-172

Elfinger M, Maucksch C, Rudolph C (2007) Characterization of lactoferrin as a targeting ligand for nonviral gene delivery to airway epithelial cells. Biomaterials 28:3448-3455

Fry DW (1978) Rapid separation of low molecular weight solutes from liposomes without dilution. J Anal Biochem 90:803-807

Gupta Y, Jain A, Jain SK (2007) Transferrin-conjugated solid lipid nanoparticles for enhanced delivery of quinine dihydrochloride to the brain. J Pharm Pharmacol 59:1-6

Ito F, Makino K (2004) Preparation and properties of monodispersed Rifampicin loaded poly(lactide-co-glycolide) microspheres. Colloids Surf B 39:17-21

Jaspart S, Piel G, Delattre L, Evrard B (2005) Solid lipid microparticles: formulation, preparation, characterisation, drug release and applications. Expert Opin Drug Deliv 2(1):75-87

Kar A (2005) Pharmaceutical drug absorption; ultraviolet and absorption methods. New Age International Publishers, India 312

Loira-Pastoriza C, Todoroff J, Vanbever R (2014) Delivery strategies for sustained drug release in the lungs. Adv Drug Deliv Rev 75:81-91

Makino K, Nakajima T, Shikamura M, Ito F, Ando S, Kochi C, Inagawa H, Soma G (2004) Efficient intracellular delivery of Rifampicin to alveolar macrophages using Rifampicin-loaded PLGA microspheres: effects of molecular weight and composition of PLGA on release of Rifampicin. Colloids Surf B 36:35-42

Muller RH, Keck CM (2010) Pharmaceutical nanoparticles-from their innovative origin to their future. Int J Pharm 390:1-3

Nassimi M, Schleh C, Lauenstein HD, Hussein R, Hoymann HG, Koch W, Pohlmann G, Krug N, Sewald K, Rittinghausen S, Braun A, Müller-Goymann C (2010) A toxicological evaluation of inhaled solid lipid nanoparticles used as a potential drug delivery system for the lung. Eur $\mathrm{J}$ Pharm Biopharm 75(2):107-116

O'Hara P, Hickey AJ (2000) Respirable PLGA microspheres containing Rifampicin for the treatment of tuberculosis: manufacture and characterization. Pharm Res 17:955-961

Pandey R, Khuller GK (2005) Solid lipid particle-based inhalable sustained drug delivery system against experimental tuberculosis. Tuberculosis 85:227-234

Pandey R, Sharma S, Khuller GK (2005) Oral solid lipid nanoparticlebased anti-tubercular chemotherapy. Tuberculosis (Edinb) 85(5-6):415-420

Sahu PK, Mishra DK, Jain N, Rajoriya V, Jain AK (2014) Mannosylated solid lipid nanoparticles for lung-targeted delivery of Paclitaxel. Drug Dev Ind Pharm (epub ahead of print)

Schubert MA, Goymann MCC (2003) Solvent injection as a new approach for manufacturing lipid nanoparticles evaluation of the method and process parameters. Eur $\mathrm{J}$ Pharm Biopharm $55: 125-131$

Sharma A, Sharma S, Khuller GK (2004) Lectin-functionalized poly (lactide-co-glycolide) nanoparticles as oral/aerosolized antitubercular drug carriers for treatment of tuberculosis. J Antimicrob Chemother 54(4):761-766

Shuhendler AJ, Prasad P, Leung M, Rauth AM, Dacosta RS, Wu XY (2012) A novel solid lipid nanoparticle formulation for active targeting to tumor $\alpha$ (v) $\beta$ (3) integrin receptors reveals cyclic RGD as a double-edged sword. Adv Healthc Mater 1(5):600-608

Tomoda K, Makino K (2007) Effects of lung surfactants on Rifampicin release rate from monodisperse Rifampicin-loaded PLGA microspheres. Colloids Surf B Biointerf 55:115-124

Tomoda K, Kojima S, Kajimoto M, Watanabe D, Nakajima T, Makino K (2005) Effects of pulmonary surfactant system on Rifampicin release from Rifampicin-loaded PLGA microspheres. Colloids Surf B Biointerf 45:1-6

Tsuda H, Sekine K, Fujita K, Ligo M (2002) Cancer prevention by bovine lactoferrin and underlying mechanisms-a review of experimental and clinical studies. Biochem Cell Biol 80:131-136

Wang M, Qin L, Li K, Zhu R, Wang W, Wang S (2012) The improvement of the anticancer effect of a novel compound benzoic acid, 2-hydroxy-, 2-D-ribofuranosylhydrazide (BHR) loaded in solid lipid nanoparticles. AAPS Pharm Sci Tech 13(4):1348-1354

Wissing SA, Muller RH (2002) Solid lipid nanoparticles as carrier for sunscreens: in vitro release and in vivo skin penetration. J Control Release 81:225-233

Yang X, Liu Y, Liu C, Zhang N (2012) Biodegradable solid lipid nanoparticle flocculates for pulmonary delivery of insulin. J Biomed Nanotech 8(5):834-842

Zhou H, Zhang Y, Biggs DL, Manning MC, Randolph TW, Christians $\mathrm{U}$, Hybertson BM, Ng K (2005) Microparticle-based lung delivery of INH decreases INH metabolism and targets alveolar macrophages. J Control Rel 107:288-299

Zhuang YG, Xu B, Huang F, Wu JJ, Chen S (2012) Solidlipidnanoparticlesof anticancer drugs against MCF-7 cell line and a murine breast cancer model. Pharmazie 67(11):925-929 